\title{
Enfermedad inflamatoria intestinal: características de fenotipo y tratamiento en un hospital universitario de Bogotá, Colombia
}

\section{Phenotypic characteristics and treatment of inflammatory bowel disease at a university hospital in Bogotá, Colombia}

Gustavo Adolfo Reyes M., ${ }^{1}$ Fabio Leonel Gil P., ${ }^{1}$ German David Carvajal P., ${ }^{1}$ Carlos Bernardo Sánchez L., ${ }^{1}$ Diego Mauricio Aponte M., ${ }^{1}$ Carlos Arturo González S., ${ }^{1}$ Johana Ibeth Gamba H., ${ }^{1}$ Javier Alberto Preciado A., ${ }^{1}$ Juan Carlos Marulanda G., ${ }^{1}$ Luis Carlos Sabbagh S. ${ }^{2}$

Departamento de Gastroenterología y Endoscopia Digestiva de la Clínica Universitaria Colombia, Fundación Universitaria Sánitas. Bogotá D. C. Colombia.

2 Jefe del servicio de Gastroenterología y Endoscopia Digestiva de la Clínica Reina Sofía. Bogotá D. C., Colombia.

Fecha recibido: 23-11-17 Fecha aceptado: $13-04-18$

\begin{abstract}
Resumen
Introducción: la enfermedad inflamatoria intestinal (EII) (colitis ulcerativa [CU], enfermedad de Crohn [EC]) es una patología inflamatoria crónica y recurrente del tracto digestivo, de causa aún incierta. La incidencia y prevalencia de esta enfermedad están en aumento a nivel mundial, especialmente en los países en vías de desarrollo. Objetivo: describir las características del fenotipo y el tipo de tratamiento administrado a 165 pacientes con diagnóstico de Ell que consultaron durante el período comprendido entre el 5 de julio del 2013 y el 31 de diciembre del 2016 en un hospital universitario de la ciudad de Bogotá, Colombia. Materiales y métodos: se realizó un estudio descriptivo basado en frecuencias y proporciones de los pacientes con diagnóstico de Ell atendidos en la Clínica Universitaria Colombia (Bogotá) entre el 5 de julio del 2013 y el 31 de diciembre del 2016. Resultados: el 75,8\% de los casos correspondió a CU y el $24,2 \%$ a EC, con un leve predominio del sexo femenino en ambas ( $56 \%$ y $55 \%$, respectivamente); la forma de presentación más frecuente de la CU fue la pancolitis en el $46,4 \%$. Los pacientes con EC tuvieron un compromiso más frecuentemente ileocolónico $(52,5 \%)$ e inflamatorio no estenosante ni fistulizante $(60 \%)$, un mayor porcentaje de manifestaciones extraintestinales (35\%), de uso de medicamentos biológicos $(35 \%)$ y requirieron un mayor número de cirugías $(27,5 \%)$ en comparación con los pacientes con CU.
\end{abstract}

Palabras clave

Enfermedad inflamatoria intestinal, colitis ulcerativa, enfermedad de Crohn.

\begin{abstract}
Inflammatory bowel disease (IBD) includes ulcerative colitis and Crohn's disease which are chronic and recurrent inflammatory pathologies of the digestive tract whose causes are still uncertain. IBD's incidence and prevalence are increasing worldwide, especially in developing countries. Objectives: The objective of this study was to describe phenotypic characteristics and types of treatment of 165 patients diagnosed with IBD treated between July 5, 2013 and December 31, 2016 at a university hospital in Bogotá, Colombia. Methods: This is a descriptive study based on frequencies and proportions of patients diagnosed with IBD at the University Clinic Colombia in Bogotá between July 5, 2013 and December 31, 2016. Results: UC accounted for 75.8\% of the cases while CD accounted for $24.2 \%$ of these cases. Women accounted for a slightly larger proportion of patients than did men: $56 \%$ of UC cases and $55 \%$ of CD cases. UC's most frequent form of presentation was pancolitis which was found $46.4 \%$ of the patients. Frequent forms of CD presentation were ileocolonic $(52.5 \%)$ and inflammatory non-stenosing or fistulizing $(60 \%)$. CD patients had a greater percentage of extraintestinal manifestations (35\%), greater use of biological medications (35\%) and required a greater number of surgeries $(27.5 \%)$ than did UC patients.
\end{abstract}

Keywords

Inflammatory bowel disease, ulcerative colitis, Crohn's disease. 


\section{INTRODUCCIÓN}

La enfermedad inflamatoria intestinal (EII) es una patología inflamatoria crónica y recurrente del tracto digestivo, de etiología incierta y, en algunos casos, con afectación de otros órganos. Se considera que la combinación de factores genéticos y medioambientales causan una alteración en la respuesta inmune que termina afectando el tracto gastrointestinal. La colitis ulcerativa (CU) y la enfermedad de Crohn (EC) son los tipos principales de EII.

La incidencia y prevalencia de la EII es mayor en los países industrializados. Sin embargo, actualmente hay una tendencia global al aumento de la incidencia y prevalencia de esta enfermedad, incluidos los países no industrializados. En 1991, en Colombia se publicó un trabajo que describía 108 casos de EII en 2 centros médicos en Bogotá ( $98 \mathrm{CU}$, 10 EC) en el período comprendido entre 1968 y 1990 (1). En 2010, se describieron a 26 pacientes en Cartagena (20 CU, 6 EC) y se estimó una prevalencia general de 29/100 000 para esta ciudad (2). En el 2010, se publicó un estudio que describió el fenotipo e historia natural de la EII en un centro de referencia en Medellín en 202 pacientes vistos entre el 2001 y el 2009; el 80,7\% correspondían a CU y el $15,8 \%$ a EC (3).

Este trabajo describe las características de fenotipo y el tipo de tratamiento administrado a 165 pacientes con EII que consultaron entre el 5 de julio del 2013 y el 31 de diciembre del 2016 en un hospital universitario de la ciudad de Bogotá, Colombia.

\section{MATERIALES Y MÉTODOS}

Se realizó un estudio descriptivo basado en frecuencias y proporciones de pacientes con diagnóstico de EII atendidos en la Clínica Universitaria Colombia (Bogotá) entre el 5 de julio del 2013 y el 31 de diciembre del 2016. Solo se incluyeron pacientes con diagnósticos confirmados de EC y CU, con base en las recomendaciones de las guías europeas para el diagnóstico de la EII $(4,5)$ y que llevaran por lo menos 3 meses de sintomatología.

\section{RESULTADOS}

- Se evaluaron a 165 pacientes, 125 con CU $(75,8 \%)$ y 40 con EC (24,2\%) (Figura 1).

- En la distribución por sexo, se encontraron 70 mujeres (56\%) y 55 hombres (44\%) en CU, y 22 mujeres (55\%) y 18 hombres (45\%) en EC (Figura 2).

- La edad promedio del diagnóstico fue de 39 años (12-75 años) en CU y de 44 años (10-82 años) en EC (Figura 3).

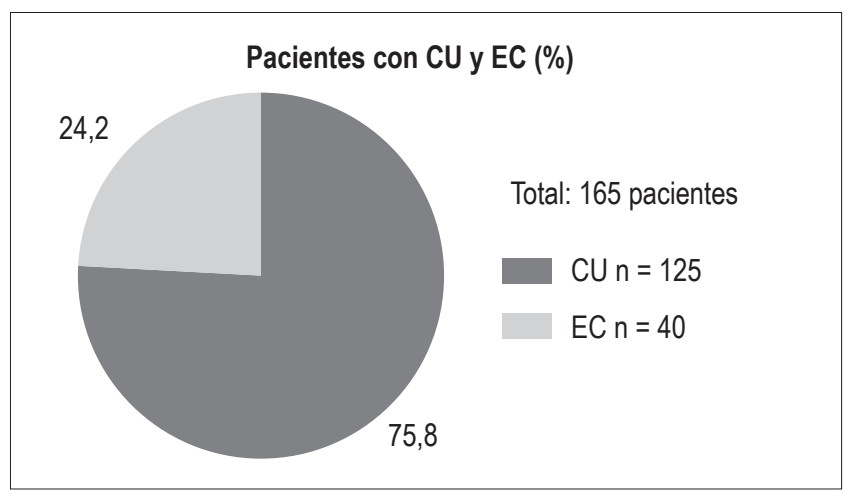

Figura 1. Porcentaje de pacientes con CU y EC.

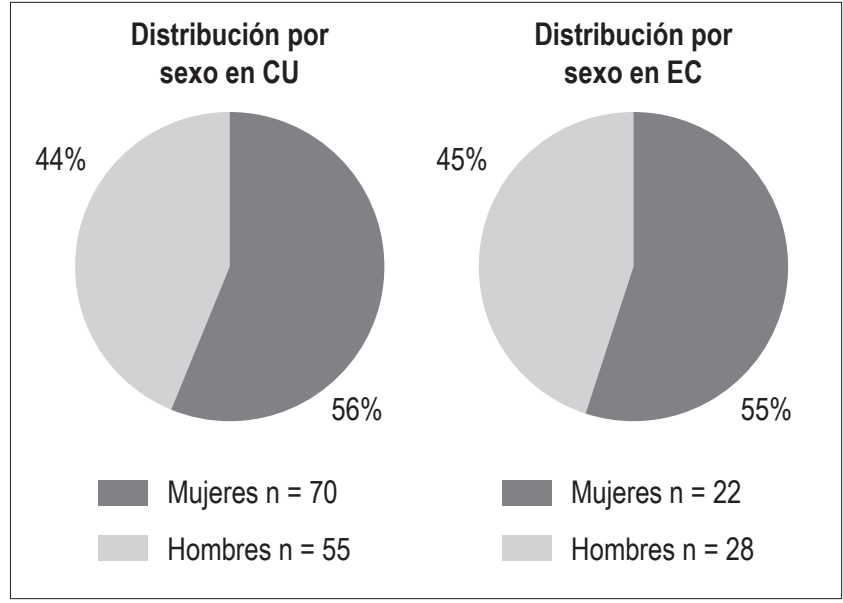

Figura 2. Distribución por sexo en CU y EC.

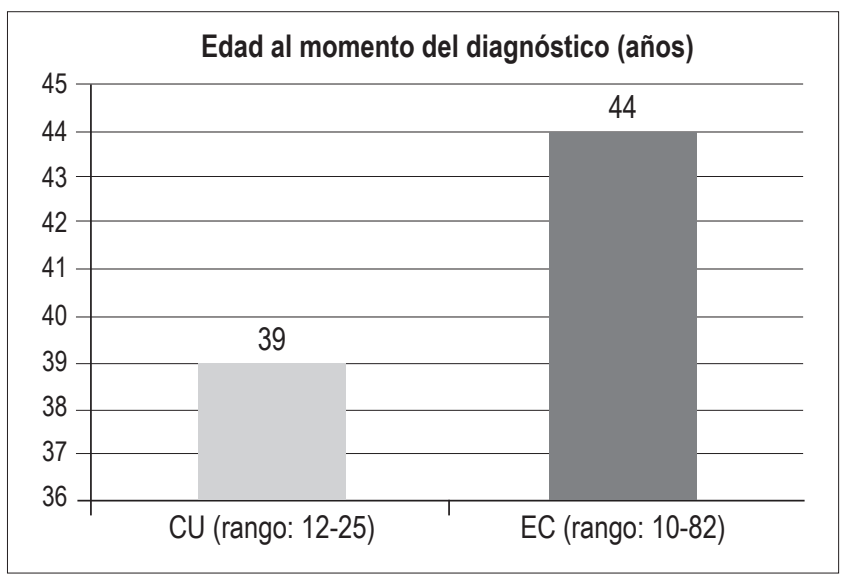

Figura 3. Edad de diagnóstico en pacientes con CU y EC.

\section{Clasificación de Montreal según la edad}

- CU (Figura 4):

- A1 (<17 años): $\mathrm{n}=5$ (4\%) 
- $\mathrm{A} 2$ (entre $17-40$ años): $\mathrm{n}=67(53,6 \%)$

- $\mathrm{A} 3$ (>40 años): $\mathrm{n}=53(42,4 \%)$

- EC (Figura 5):

- $\mathrm{A} 1$ (<17 años): $\mathrm{n}=1(2,5 \%)$

- $\mathrm{A} 2$ (entre $17-40$ años): $\mathrm{n}=16(40 \%)$

- $\mathrm{A} 3$ (>40 años): $\mathrm{n}=23(57,5 \%)$.

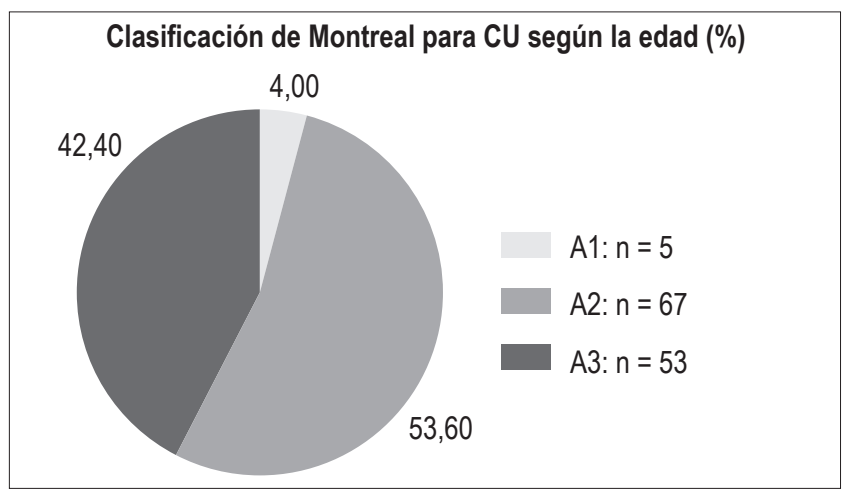

Figura 4. Clasificación de Montreal para CU según la edad.

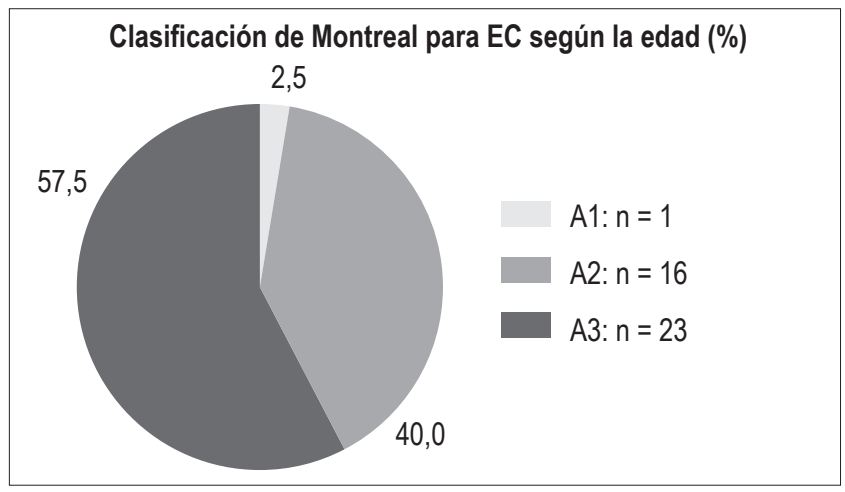

Figura 5. Clasificación de Montreal para EC según la edad.

\section{Extensión de la enfermedad (clasificación de Montreal) (Figura 6)}

- CU:

- $\mathrm{E} 1$ (proctitis): $\mathrm{n}=26(20,8 \%)$

- E2 (colitis izquierda): $\mathrm{n}=41(32,8 \%)$

- E3 (pancolitis): $\mathrm{n}=58(46,4 \%)$

- EC:

- L1 (íleon): $\mathrm{n}=14(35 \%)$

- L2 (colon): $\mathrm{n}=5(12,5 \%)$

- L3 (ileocolónica): $\mathrm{n}=21(52,5 \%)$

- L4 (tracto digestivo superior aislado): $\mathrm{n}=0$.

\section{Comportamiento de la EC (Figura 7)}

- B1 (inflamatorio): $\mathrm{n}=24(60 \%)$
- B2 (estenosante): $\mathrm{n}=13(32,5 \%)$

- B3 (penetrante fistulizante, diferente a perianal): $\mathrm{n}=1$ $(2,5 \%)$

- $\mathrm{P}($ perianal $): \mathrm{n}=2(5 \%)$.

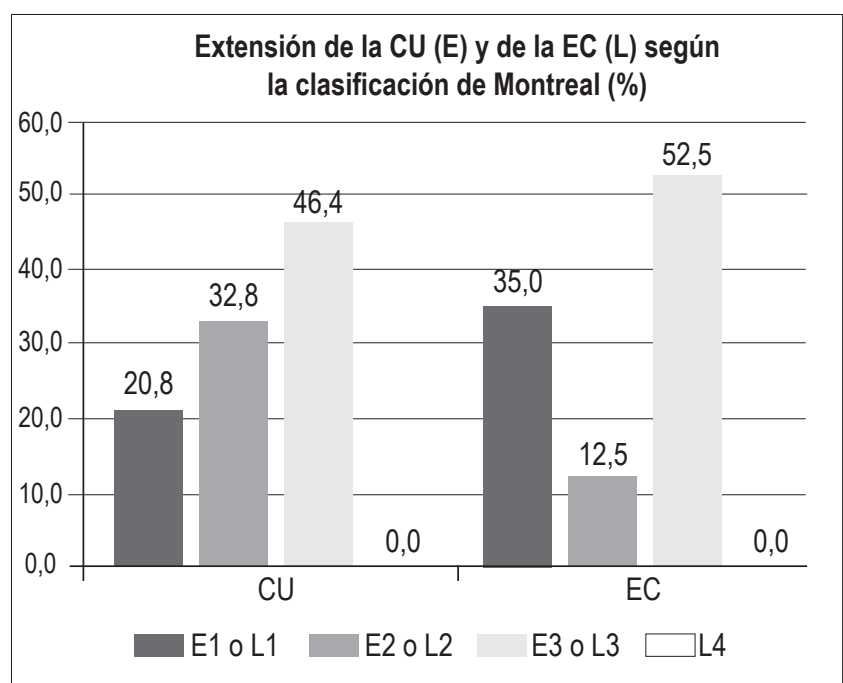

Figura 6. Extensión de la CU y de la EC según la clasificación de Montreal.

Clasificación de Montreal según el comportamiento de la EC (\%)

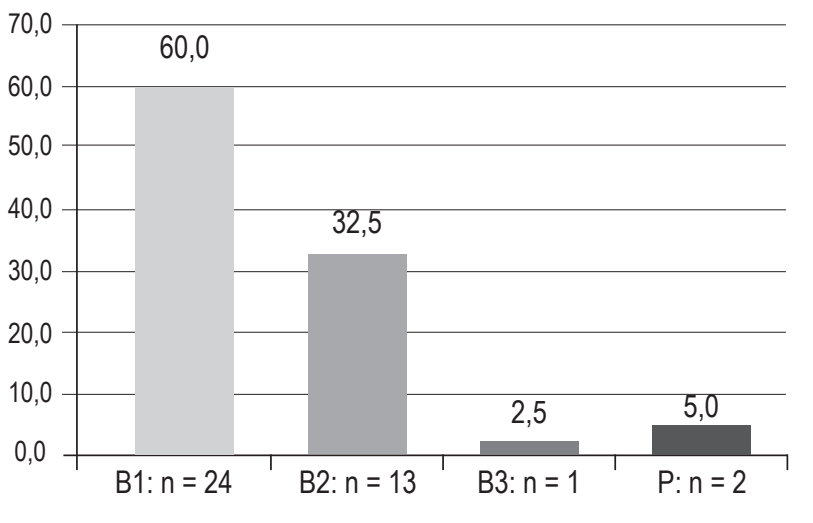

Figura 7. Clasificación de Montreal según el comportamiento de la EC.

\section{Manifestaciones extraintestinales}

- En pacientes con CU (Figura 8): 31 casos $(24,8 \%)$ distribuidos así:

- Articulares: 29 casos;

- Oculares: 4 .

- Aftas orales: 4;

- Colangitis esclerosante: 3 ; 
- Dermatológicas (eritema nodoso, pioderma gangrenoso): 3 ;

- Trombosis venosa: 2;

- Retraso del crecimiento: 1.3 pacientes tenían más de 1 manifestación extraintestinal.

- En pacientes con EC (Figura 9): 14 casos (35\%) distribuidos así:

- Articulares: 8;

- Aftas orales: 8 ;

- Oculares: 2;

- Dermatológicas: 2 (dermatitis herpetiforme, pioderma gangrenoso);
- Colangitis esclerosante: 1 ;

- Trombosis venosa: 1.8 pacientes tenían más de 1 manifestación extraintestinal.

\section{Tratamiento para los pacientes con CU (Figura 10)}

- Sin tratamiento: $16(12,8 \%)$

- Aminosalicilatos: $104(83,2 \%)$ :

- Solo oral: $53(42,4 \%)$

- Oral y rectal: $51(40,8 \%)$

- Inmunomoduladores:

- Azatioprina, mercaptopurina: 29 (23,2\%)

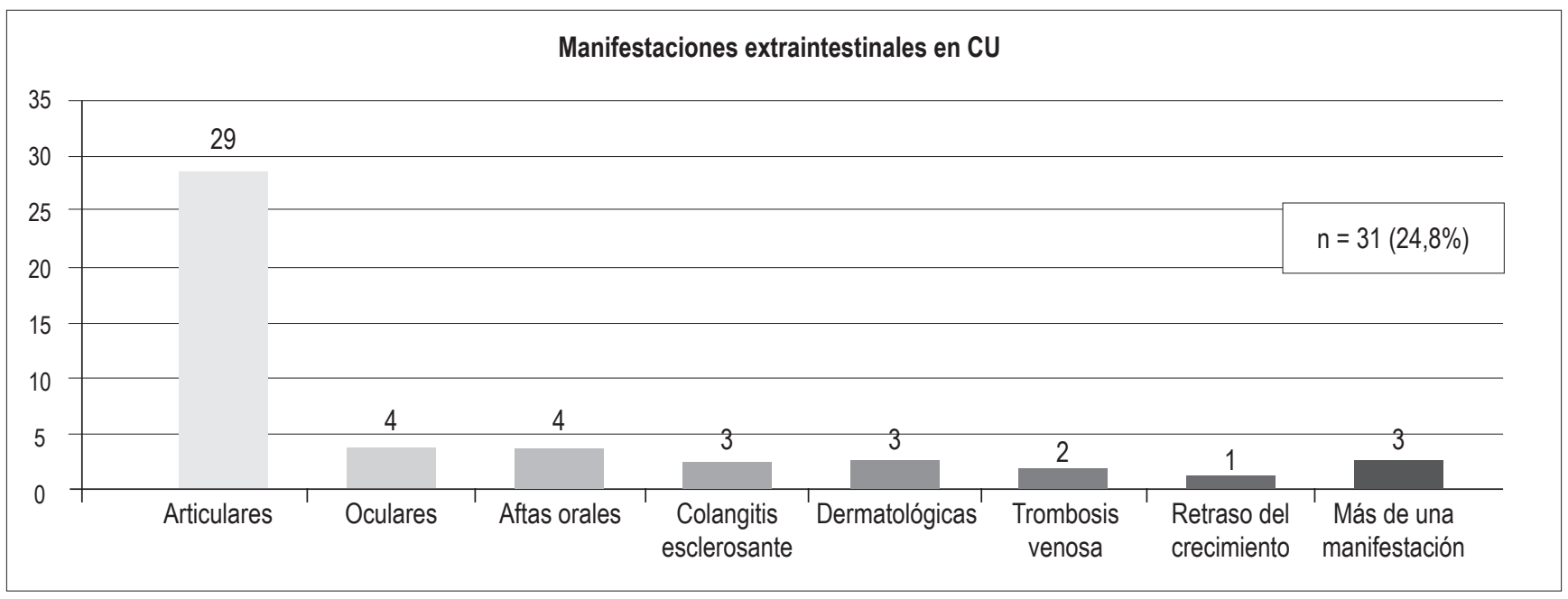

Figura 8. Distribución de las manifestaciones extraintestinales en la CU.

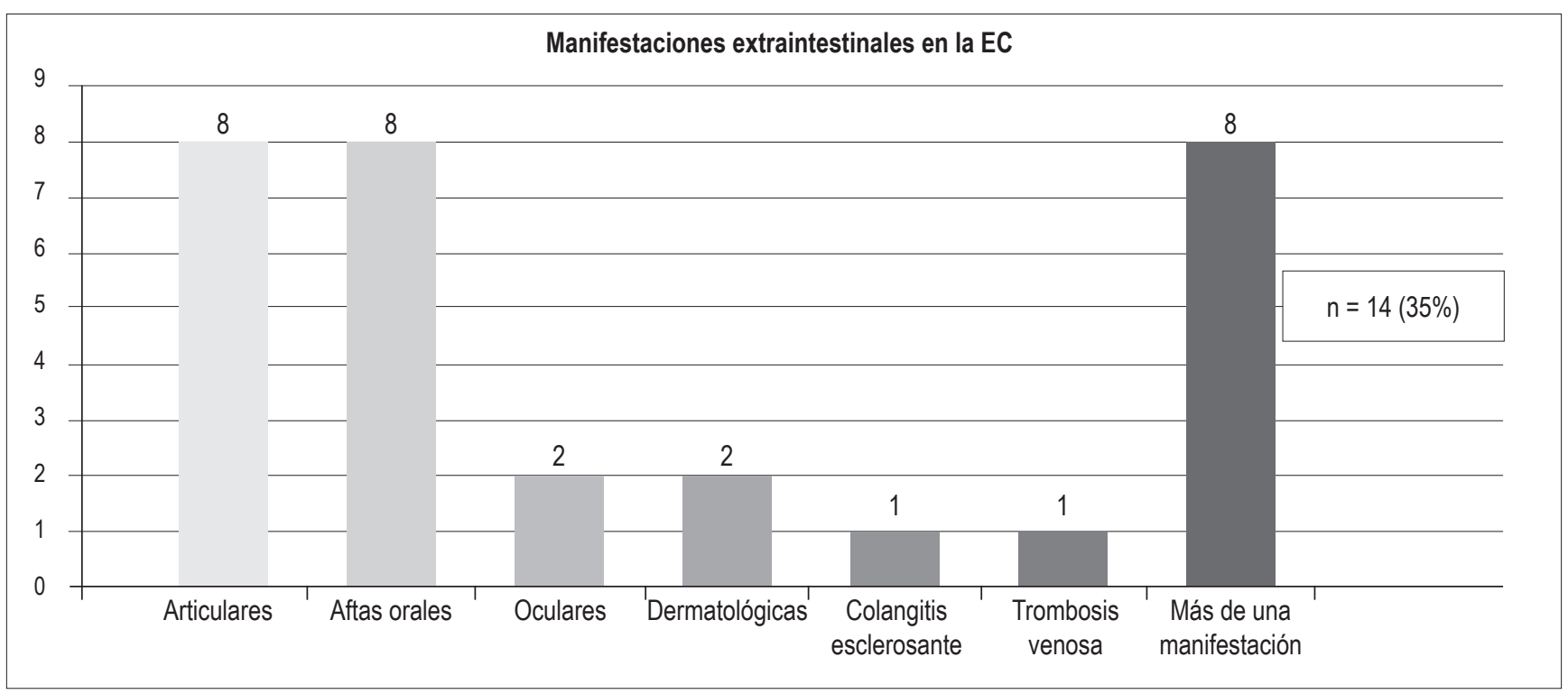

Figura 9. Distribución de las manifestaciones extraintestinales en la EC. 


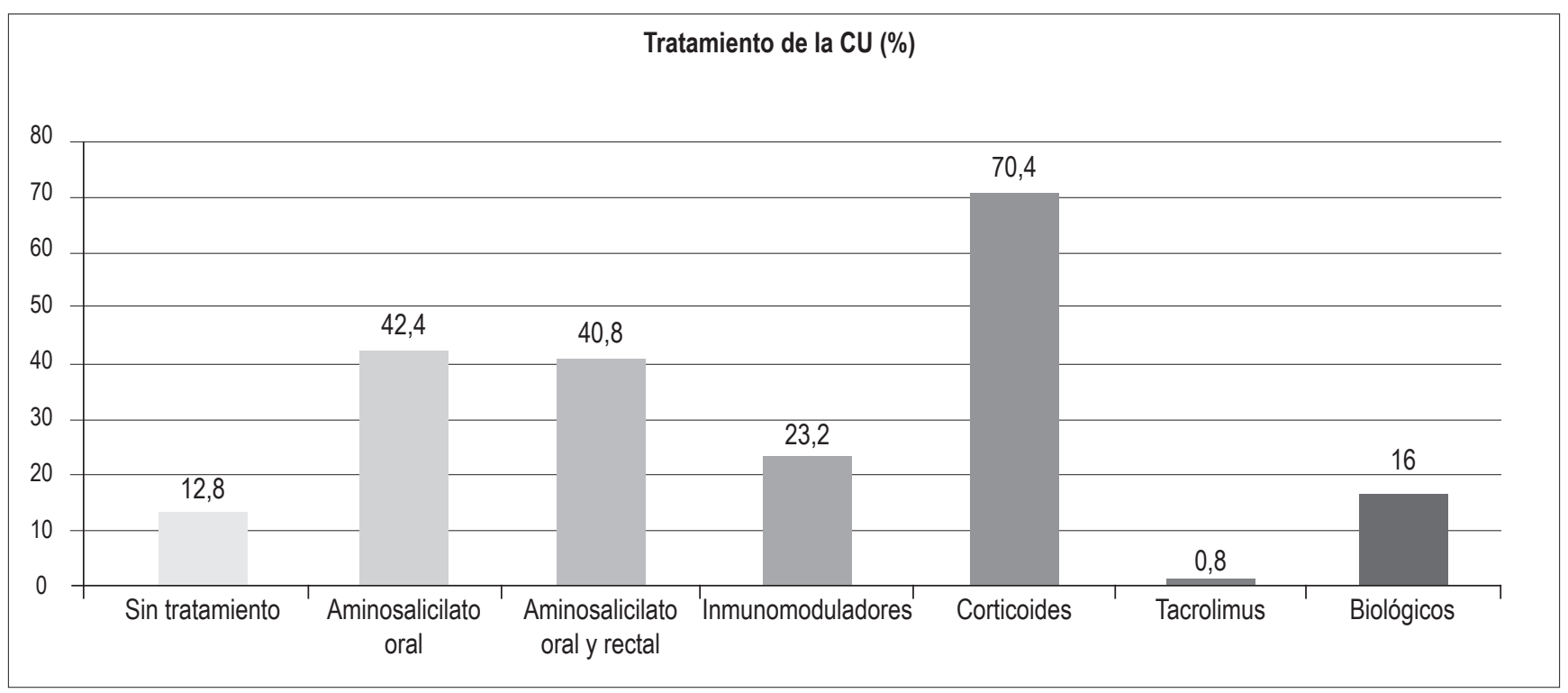

Figura 10. Tratamientos realizados en los pacientes con CU.

- Exposición a corticoides: $88(70,4 \%)$

- Tacrolimus: $1(0,8 \%)$

- Biológicos: 20 (16\%):

- Infliximab: 13

- Adalimumab: 6

- Vedolizumab: 1. El 44\% de los pacientes con biológicos recibieron terapia combinada con inmunomodulador.

\section{Tratamiento para los pacientes con EC (Figura 11)}

- Aminosalicilatos: $12(30 \%)$

- Inmunomoduladores: $14(35 \%)$

- Exposición a corticoides: $14(35 \%)$

- Biológicos: 14 pacientes (35\%):

- Adalimumab: 6

- Infliximab: 6

- Vedolizumab: 2. De los pacientes en tratamiento con biológico, $4(28,5 \%)$ estaban bajo terapia combinada con inmunomodulador. 1 paciente recibió tacrolimus $(2,5 \%)$.

\section{Cirugías}

- En pacientes con CU: 6 pacientes $(4,8 \%)$ requirieron colectomía total (Figura 12).

- En pacientes con EC: 11 pacientes (27,5\%):

- Resecciones intestinales: 2

- Abscesos y fístulas perianales: 2

- Obstrucción intestinal: 2

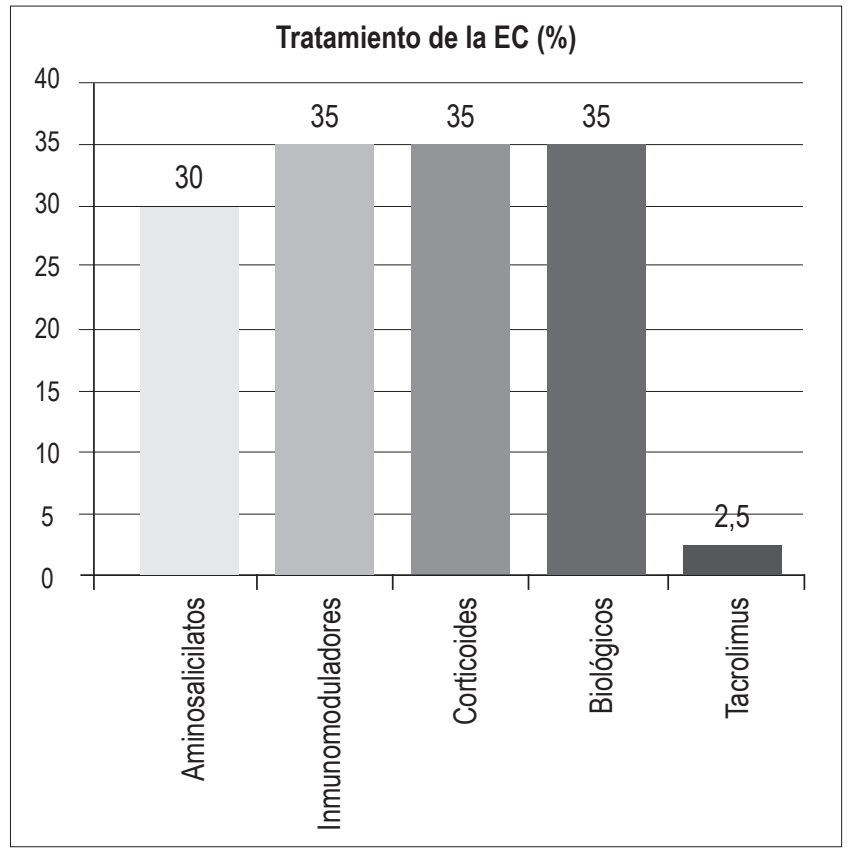

Figura 11. Tratamientos realizados en los pacientes con EC.

- Fístulas enterocutáneas: 1 (Figuras 13 y 14).

\section{DISCUSIÓN}

La EII es más común en países desarrollados, especialmente en Norteamérica y Europa occidental (6). La incidencia 


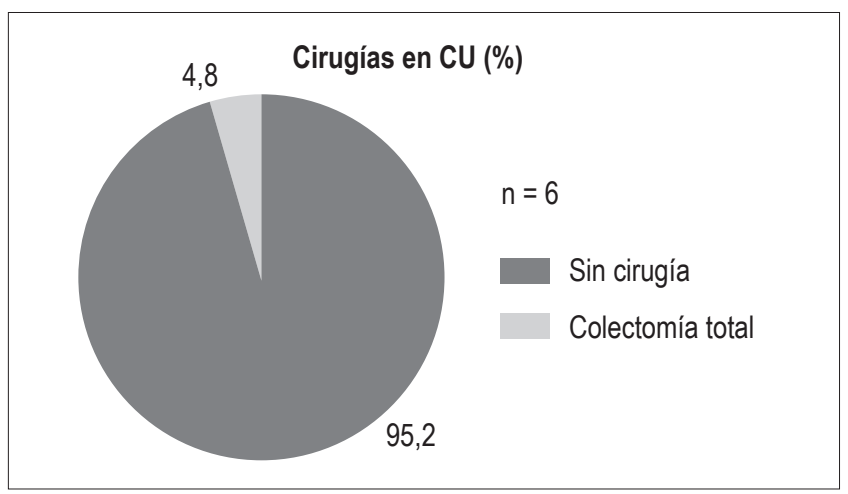

Figura 12. Porcentaje de cirugías realizadas en pacientes con CU.

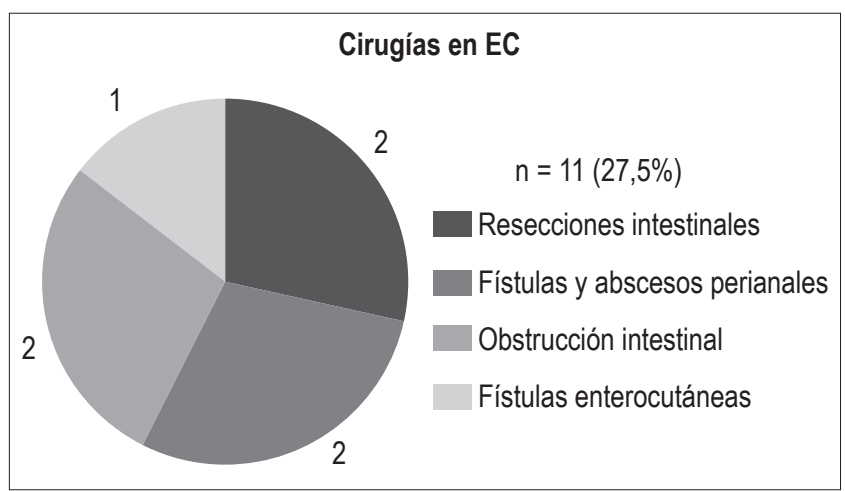

Figura 14. Tipos de cirugías realizadas en los pacientes con EC.

anual de la CU en Norteamérica es de 19 casos por 100000 habitantes y la prevalencia es de 37-248 casos por 100000 habitantes. La incidencia en Europa es de 24 casos por 100000 habitantes, con una prevalencia de 4,9-505 casos por 100000 habitantes. La incidencia de la EC es similar: 20 casos por 100000 habitantes en Norteamérica y 12,7 casos por 100000 habitantes en Europa (7). La incidencia de la EII se ha incrementado a nivel mundial. En Europa, la incidencia de la CU aumentó de 6 casos por 100000 personas/año y la de EC de 1 caso por 100000 personas/ año en 1962 a 9,8 y 6,3 casos por 100000 personas/año respectivamente en el 2010 (8). Similares resultados se han observado en Estados Unidos (9). La EII era considerada infrecuente en los países orientales; sin embargo, los datos epidemiológicos en Japón, Corea y Hong Kong han mostrado una incidencia aumentada entre 1980 y 2003 (10). En estas poblaciones, la incidencia de la CU es mayor que la de la EC y el aumento en la incidencia de la CU casi siempre precede al aumento de la incidencia de la EC por una década (7), hallazgos que pudieran ser similares a lo que está ocurriendo en la población colombiana. Se considera

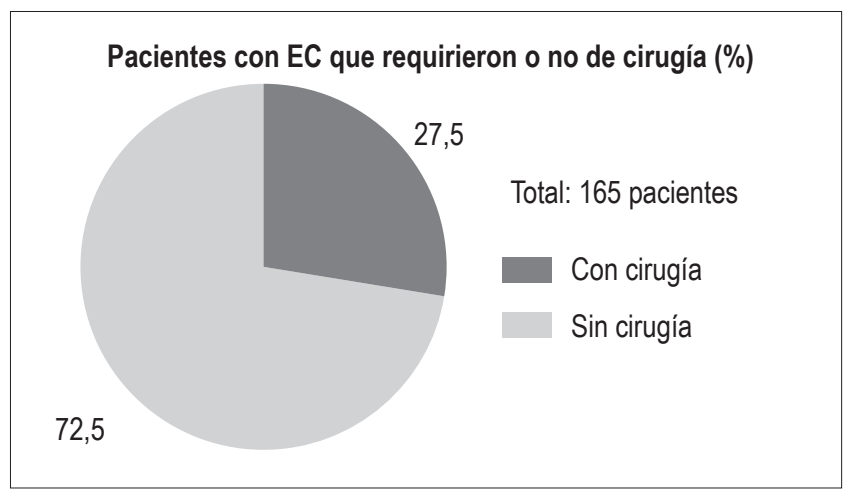

Figura 13. Porcentaje de pacientes con EC que requirieron de cirugía.

que la occidentalización de los hábitos de vida es un factor que puede influir en el aumento en la incidencia de la EII (6).

Este estudio describe a 165 pacientes con EII pertenecientes a una entidad prestadora de salud que maneja medicina del plan obligatorio de salud y prepagada, valorados en la Clínica Universitaria Colombia en Bogotá. El 75,8\% de los casos correspondieron a CU y el $24,2 \%$ a EC, lo cual concuerda con otros estudios realizados en Colombia en los que se observa que la CU es más frecuente (1-3).

El sexo femenino correspondió al 56\% en la CU y al 55\% en la EC. En otros estudios se ha encontrado una relación mujer: hombre de 1,3:1 para la EC y sin diferencias de sexo para la CU (11-13).

La CU puede comenzar a cualquier edad, aunque es infrecuente antes de los 5 años y después de los 75 años. La incidencia pico sucede en la segunda y tercera décadas de vida, con un segundo pico entre los 60 y 70 años. La EC se presenta con mayor frecuencia entre los 15-30 años, con una edad promedio de 30 años, aunque se ha observado un aumento en el diagnóstico de la EC en pacientes de 60 años $y$ mayores $(14,15)$. En este estudio, la edad promedio en que se hizo el diagnóstico fue de 39 años (12-75 años) para CU y de 44 años (10-82 años) para EC; la mayoría de los pacientes con CU correspondieron al grupo A2 (53\%) y en la EC al grupo A3 (57,5\%), lo que demuestra una presentación más frecuente en el grupo de mayor edad para la EC, hallazgos similares a los de Juliao (3). Debido a que este estudio se basó en una población atendida por un servicio de gastroenterología para adultos, es de esperar que los grupos A1 (menores de 17 años) tanto para CU como para EC estén subvalorados.

El fenotipo de la EII ha cambiado en los últimos años $(16,17)$. Aunque la extensión de la CU se divide en forma relativamente equivalente entre proctitis, colitis izquierda y pancolitis, la proporción de pacientes que se presentan con pancolitis se ha incrementado en las últimas décadas 
en países como Dinamarca, donde la pancolitis correspondía al 18\% de los casos entre 1962-1987 y se incrementó al 27\% entre el 2003-2004 (18). En Australia hay predominio de pancolitis: $41 \%$ (19). La colitis izquierda es la manifestación inicial más frecuente en Hungría: 50\% (20) y Holanda: $52 \%$ (21), y la proctitis es la más frecuente en Asia: 37\% (19) (Figura 15). En estudios previos en la población colombiana (3) se ha encontrado predominio de la colitis izquierda (45\%), mientras que en este estudio la principal manifestación fue pancolitis: $46,4 \%$; y la proctitis fue la menos frecuente: $20,8 \%$.

Con respecto a la EC, el compromiso ileocolónico fue el más frecuente (52,5\%), similar a los hallazgos en estudios de Hungría, Holanda, Australia-Asia (Figura 16) y Colombia $(50 \%)(3,19-21)$. Se encontró un compromiso aislado de íleon en el 35\%, aislado de colon en el 12,5\% y

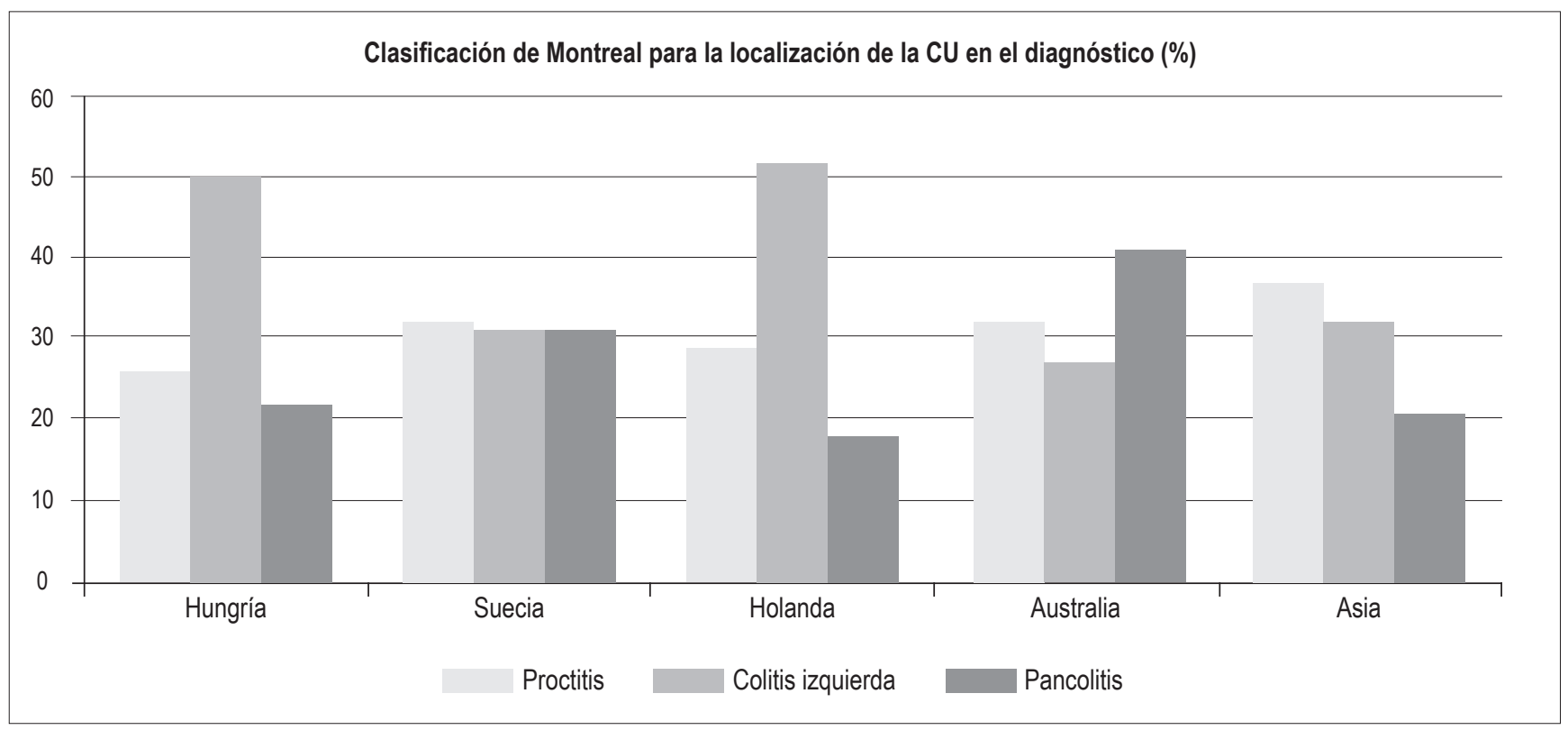

Figura 15. Clasificación de Montreal para la localización de la CU en el diagnóstico. Modificado de: Moran C et al. Gastroenterol Res Pract. 2016;2016:1-9.

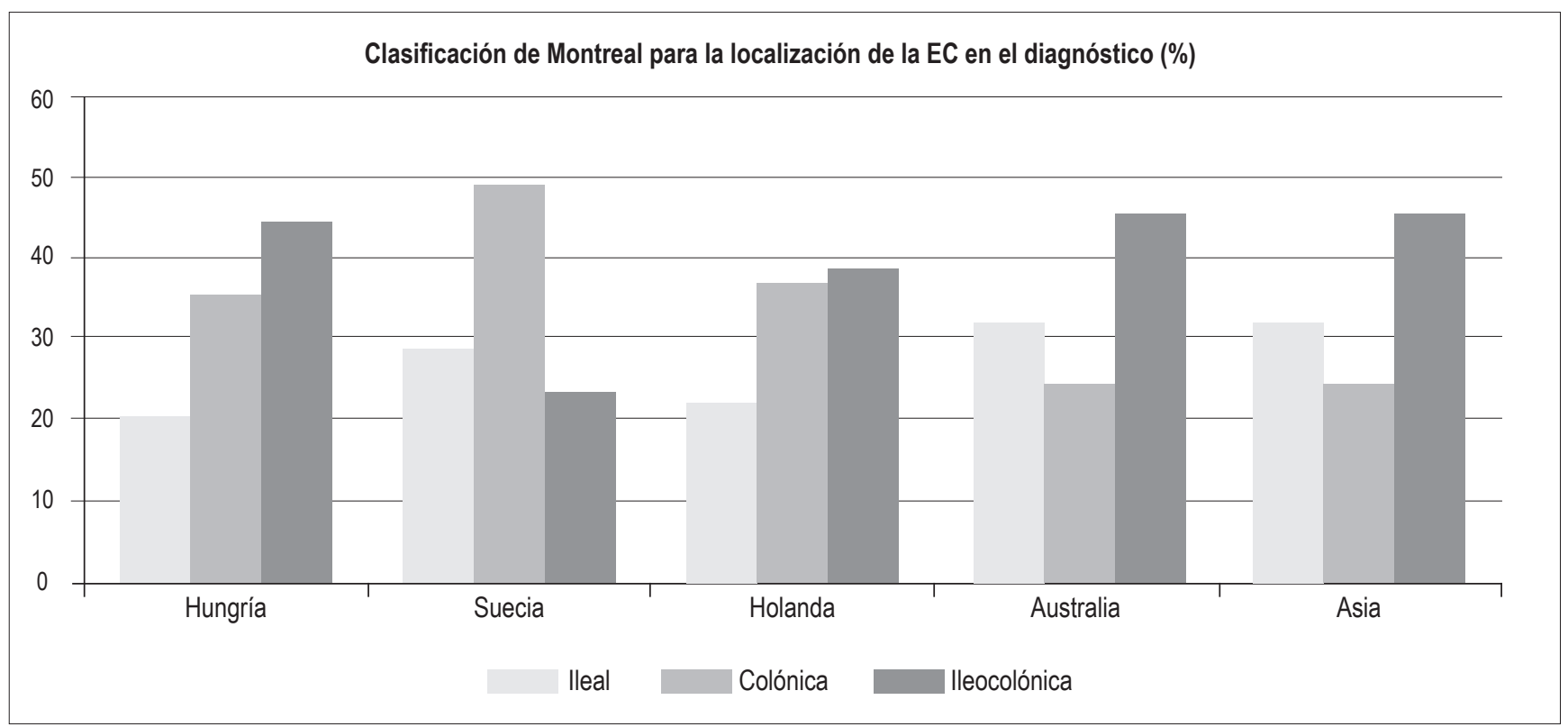

Figura 16. Clasificación de Montreal para la localización de la EC en el diagnóstico. Modificado de: Moran C et al. Gastroenterol Res Pract. 2016;2016:1-9. 
no se encontraron casos de EC de tracto digestivo alto. El comportamiento de la EC fue principalmente inflamatorio $(60 \%)$, seguido por un fenotipo estenosante $(32,5 \%)$, perianal $(5 \%)$ y fistulizante no perianal (2,5\%). Este comportamiento es similar al encontrado en un estudio en Holanda, donde la mayoría de los casos fue de tipo inflamatorio luminal (68\%), seguido por el fenotipo estenosante (19\%) con una menor proporción de manifestaciones fistulizantes y perianal ( $11 \%$ y $9 \%$, respectivamente). Hallazgos similares se han encontrado en un estudio multicéntrico europeo y en Noruega, con predominio del fenotipo inflamatorio en el $73,7 \%$ y $62 \%$, respectivamente; seguidos por el fenotipo estenosante $(15,9 \%$ y $27 \%$, respectivamente $)(22,23)$. En contraste, el estudio del Dr. Juliao (3) en la población colombiana encontró un porcentaje menor de fenotipo inflamatorio $(34,4 \%)$, probablemente por el lapso prolongado entre el inicio de la sintomatología y la consulta.

Un 50\% de los pacientes con EII presentan al menos una manifestación extraintestinal, más frecuentemente en la EC (especialmente con compromiso colónico), en comparación con la CU (24). En el presente estudio se encontró que un mayor porcentaje de pacientes con EC (35\%) presentó manifestaciones extraintestinales en comparación con los pacientes con CU $(24,8 \%)$; las más frecuentes fueron las articulares en ambos grupos (23\% en la CU y $20 \%$ en la EC) y las aftas orales (3,2\% en la CU y $20 \%$ en la EC). Estos resultados son similares a los encontrados en la población colombiana y en otros grupos, donde se reportan manifestaciones articulares en un 16\%-35\% de los pacientes (3, 25-27). Un mayor porcentaje de pacientes con EC (20\%) presentó más de una manifestación extraintestinal; esto sucedió en 3 pacientes con CU $(2,4 \%)$.

El tratamiento de la $\mathrm{CU}$ depende de varios factores (28). La terapia de primera línea son los aminosalicilatos. En este estudio, el 100\% de los pacientes recibió en algún momento aminosalicilatos. Por ser un estudio descriptivo, al momento de recolectar la información, el 83,2\% de los pacientes recibieron aminosalicilatos $(42,4 \%$ por vía oral y $40,8 \%$ por vías oral y rectal). Un $12,8 \%$ no recibió aminosalicilatos debido principalmente a la falta de eficacia del medicamento o al antecedente de colectomía total. El $23,2 \%$ de los pacientes recibió un inmunomodulador (azatioprina o 6-mercaptopurina). La exposición a corticoides se observó en el 70,4\% de los pacientes y en ningún caso se utilizaron de forma crónica. En los casos refractarios a la terapia inicial (aminosalicilatos e inmunomoduladores), en los pacientes dependientes o refractarios a corticosteroides y en los pacientes hospitalizados con una colitis activa grave que no responde en 3 a 5 días al uso de corticoides endovenosos se indicó el uso de terapia biológica (28). El $16 \%$ de los pacientes con CU recibió tratamiento biológico y la mayoría cursaba con pancolitis (75\%), el 20\% corres- pondió a E2 y el 5\% a E1. El biológico más frecuentemente administrado fue el infliximab (13 pacientes). 6 pacientes recibieron adalimumab y 1 paciente vedolizumab (Figura 17). El $44 \%$ de los pacientes con biológicos recibió concomitantemente un inmunomodulador: $61,5 \%$ de los pacientes con infliximab y $16,6 \%$ de los pacientes con adalimumab. La principal razón para que los pacientes con infliximab no recibieran inmunomodulador fue la presencia de efectos adversos (dolor abdominal, elevación de enzimas hepáticas o toxicidad hematológica). 1 paciente $(0,8 \%)$ con pancolitis recibió tacrolimus debido a la falta de respuesta al infliximab y 1 paciente recibió vedolizumab como tercer biológico, por falta de respuesta a los 2 antifactor de necrosis tumoral (anti-TNF) y haber rechazado la posibilidad de colectomía.

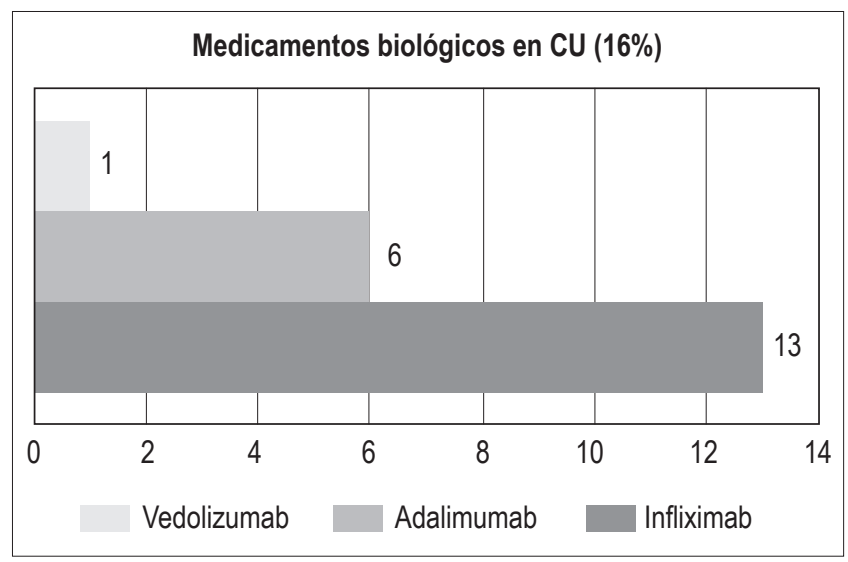

Figura 17. Medicamentos biológicos usados para el tratamiento de la CU.

Para el tratamiento de la EC existen varias alternativas: corticoides, inmunomoduladores y biológicos (29). La mesalazina ha mostrado un beneficio muy marginal en la EC. En un estudio se encontró que la mesalazina era equivalente a la budesonida en la enfermedad ileocecal leve, lo cual podría sugerir un rol para los aminosalicilatos en la EC leve; sin embargo, un metaanálisis confirmó que la budesonida debe preferirse en este escenario clínico. No se ha encontrado una clara evidencia de que la mesalazina sea mejor que el placebo en la EC (29). En este estudio, el 30\% de los pacientes con EC recibió aminosalicilatos, el 35\% estuvo expuesto a corticoides ( $21 \%$ budesonida y $79 \%$ corticoides sistémicos) y un 35\% recibió inmunomoduladores (azatioprina o 6-mercaptopurina). En comparación con los pacientes con $\mathrm{CU}$, un mayor porcentaje de pacientes con EC (35\%) requirió un tratamiento con biológicos, siendo igual la frecuencia del uso de infliximab y adalimumab (6 pacientes para cada medicamento) (Figura 18). 2 pacientes recibieron vedolizumab: una paciente con intolerancia a infliximab (leucopenia severa) y no respondedora primaria a adalimumab, y un paciente con pérdida de respuesta a infliximab y no respon- 
dedor primario a adalimumab. El 28,5\% de los pacientes con anti-TNF recibió terapia combinada con un inmunomodulador. 1 paciente $(2,5 \%)$ recibió tacrolimus.

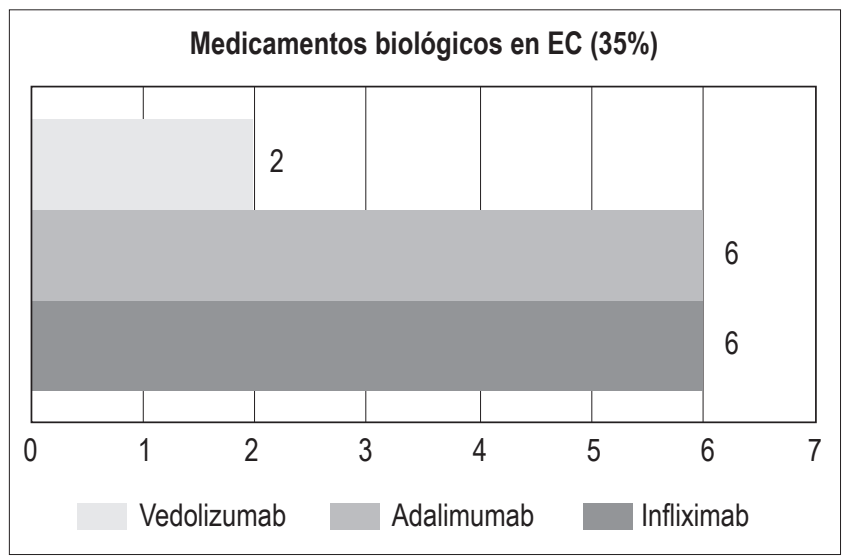

Figura 18. Medicamentos biológicos usados para el tratamiento de EC.

Entre un 4\%-9\% de los pacientes con CU requiere cirugía (proctocolectomía) como tratamiento definitivo durante el primer año del diagnóstico y el riesgo de necesitar cirugía después es del $1 \%$ por año. Las indicaciones de cirugía pueden ser urgentes (perforación de colon, megacolon tóxico, colitis fulminante refractaria al manejo médico y sangrado no controlado) o electivas (refractariedad al manejo médico, adenocarcinoma de colon y algunos casos de hallazgo de displasia en las biopsias) (30). En este estudio, el 4,8\% de los pacientes con CU requirió colectomía total, similar a los datos publicados en otros países y en Colombia (6\% en el estudio de Juliao) (3).

A pesar de los avances en el tratamiento médico de la EC, un porcentaje significativo de los pacientes aún necesita alguna cirugía. Un $80 \%$ de los pacientes puede requerir al menos un procedimiento quirúrgico a lo largo de su vida, con un riesgo alto de recurrencia posquirúrgica (30\% a los 3 años y $60 \%$ a los 10 años). El $70 \%$ de los pacientes puede necesitar una segunda resección intestinal (31). Las indicaciones de cirugía en EC son variadas: enfermedad obstructiva sin actividad inflamatoria significativa, abscesos, fístulas, la indicación de estenoplastia y la enfermedad perianal $(31,32)$. En este estudio, un $27,5 \%$ de los pacientes con EC requirió algún tipo de cirugía: resecciones intestinales por estenosis y masa $(\mathrm{n}=2)$, abscesos y fístulas perianales ( $\mathrm{n}$ $=2$ ), obstrucción intestinal sin requerimiento de resección intestinal $(\mathrm{n}=2)$ y fístula enterocutánea $(\mathrm{n}=1)$.

\section{REFERENCIAS}

1. Argüello M, Archila PE, Sierra F, et al. Enfermedad inflamatoria intestinal. Rev Col Gastroenterol. 1991;6(4):237-72.
2. Yepes I, Carmona R, Díaz F, et al. Prevalencia y características demográficas de la enfermedad inflamatoria intestinal en Cartagena, Colombia. Rev Col Gastroenterol. 2010;25(2):107-11.

3. Juliao F, Ruiz M, Flórez J, et al. Fenotipo e historia natural de la enfermedad inflamatoria intestinal en un centro de referencia en Medellín, Colombia. Rev Col Gastroenterol. 2010;25(3):240-51.

4. Van Assche G, Dignass A, Panés J, et al. The second European evidence-based Consensus on the diagnosis and management of Crohn's disease: Definitions and diagnosis. J Crohns Colitis. 2010;4(1):63-101. doi: 10.1016/j.crohns.2009.09.009.

5. Dignass A, Eliakim R, Magro F, et al. Second European evidence-based consensus on the diagnosis and management of ulcerative colitis part 1: definitions and diagnosis. J Crohns Colitis. 2012;6(10):965-90. doi: 10.1016/j.crohns.2012.09.003.

6. Hanauer S. Inflammatory bowel disease: epidemiology, pathogenesis and therapeutic opportunities. Inflamm Bowel Dis. 2006; 12 Suppl 1:S3-9.

7. Ananthakrishnan AN. Epidemiology and risk factors for IBD. Nat Rev Gastroenterol Hepatol. 2015;12(4):205-17. doi: $10.1038 /$ nrgastro.2015.34.

8. Burisch J, Munkholm P. The epidemiology of inflammatory bowel disease. Scand J Gastroenterol. 2015;50(8):942-5. doi: 10.3109/00365521.2015.1014407.

9. Loftus EV Jr, Silverstein MD, Sandborn WJ, et al. Ulcerative colitis in Olmsted County, Minnesota, 1940-1993: incidence, prevalence, and survival. Gut. 2000;46(3):336-43. doi: 10.1136/gut.46.3.336.

10. Thia KT, Loftus EV Jr, Sandborn WJ, et al. An update on the epidemiology of inflammatory bowel disease in Asia. Am J Gastroenterol. 2008;103(12):3167-82. doi: 10.1111/j.1572-0241.2008.02158.x.

11. Kappelman MD, Rifas-Shiman SL, Kleinman K, et al. The prevalence and geographic distribution of Crohn's disease and ulcerative colitis in the United States. Clin Gastroenterol Hepatol. 2007;5(12):1424-9. doi: 10.1016/j. cgh.2007.07.012.

12. Jacobsen BA, Fallingborg J, Rasmussen $\mathrm{HH}$, et al. Increase in incidence and prevalence of inflammatory bowel disease in northern Denmark: a population-based study, 1978-2002. Eur J Gastroenterol Hepatol. 2006;18(6):601-6.

13. Osterman M, Lichtenstein G. Ulcerative colitis. En: Feldman M, Friedman LS, Brandt LJ (editores). Sleisenger and Fordtran's gastrointestinal and liver diseases. 10. ${ }^{a}$ edición. Elsevier; 2015. pp. 2023-61.

14. Loftus CG, Loftus EV Jr, Harmsen WS, et al. Update on the incidence and prevalence of Crohn's disease and ulcerative colitis in Olmsted County, Minnesota, 1940-2000. Inflamm Bowel Dis. 2007;13(3):254-61. doi: 10.1002/ibd.20029.

15. Bernstein CN, Wajda A, Svenson LW, et al. The epidemiology of inflammatory bowel disease in Canada: a population-based study. Am J Gastroenterol. 2006;101(7):155968. doi: 10.1111/j.1572-0241.2006.00603.x. 
16. Langholz E, Munkholm P, Davidsen M, et al. Course of ulcerative colitis: Analysis of changes in disease activity over years. Gastroenterology. 1994;107(1):3-11. doi: 10.1016/0016-5085(94)90054-X.

17. Moran C, Sheehan D, Shanahan F. The changing phenotype of inflammatory bowel disease. Gastroenterol Res Pract. 2016;2016:1-9. doi: 10.1155/2016/1619053.

18. Jess T, Riis L, Vind I, et al. Changes in clinical characteristics, course, and prognosis of inflammatory bowel disease during the last 5 decades: a population-based study from Copenhagen, Denmark. Inflamm Bowel Dis. 2007;13(4):481-9. doi: 10.1002/ibd.20036.

19. Ng SC, Tang W, Ching JY, et al. Incidence and phenotype of inflammatory bowel disease based on results from the Asia-Pacific Crohn's and colitis epidemiology study. Gastroenterology. 2013;145(1):158-65.e2. doi: 10.1053/j. gastro.2013.04.007.

20. Lakatos L, Kiss LS, David G, et al. Incidence, disease phenotype at diagnosis, and early disease course in inflammatory bowel diseases in Western Hungary, 2002-2006. Inflamm Bowel Dis. 2011;17(12):2558-65. doi: 10.1002/ibd.21607.

21. Nuij VJ, Zelinkova Z, Rijk MC, et al. Phenotype of inflammatory bowel disease at diagnosis in the Netherlands: a population-based inception cohort study (the Delta Cohort) Inflamm Bowel Dis. 2013;19(10):2215-22. doi: 10.1097/MIB.0b013e3182961626.

22. Wolters FL, Russel MG, Sijbrandij J, et al. Phenotype at diagnosis predicts recurrence rates in Crohn's disease. Gut 2006;55(8):1124-30. doi: 10.1136/gut.2005.084061.

23. Solberg IC, Vatn MH, Høie O, et al. Clinical course in Crohn's disease: results of a Norwegian population-based ten-year follow-up study. Clin Gastroenterol Hepatol. 2007;5(12):1430-8. doi: 10.1016/j.cgh.2007.09.002.

24. Harbord M, Annese V, Vavricka S, et al. The first european evidence-based consensus on extra-intestinal manifes- tations in inflammatory bowel disease. J Crohns Colitis. 2016;10(3):239-54. doi: 10.1093/ecco-jcc/jjv213.

25. Orchard TR, Wordsworth BP, Jewell DP. Peripheral arthropathies in inflammatory bowel disease: their articular distribution and natural history. Gut. 1998;42(3):387-91. doi: 10.1136/gut.42.3.387.

26. Kethu S. Extraintestinal manifestations of inflammatory bowel diseases. J Clin Gastroenterol, 2006;40(6):467-75. doi: 10.1097/00004836-200607000-00003.

27. Danese S, Semeraro S, Para A, et al. Extraintestinal manifestations in inflammatory bowel disease. World J Gastroenterol. 2005;11(46):7227-36. doi: 10.3748/wjg.v11.i46.7227.

28. Dignass A, Lindsay J, Sturm A, et al. Second European evidence-based consensus on the diagnosis and management of ulcerative colitis Part 2: Current management. J Crohns Colitis. 2012;6(10):991-1030. doi: 10.1016/j.crohns.2012.09.002.

29. Gomollón F, Dignass A, Annese V, et al. 3rd European evidence-based consensus on the diagnosis and management of Crohn's disease 2016: Part 1: Diagnosis and medical management. J Crohns Colitis. 2017;11(1):3-25. doi: 10.1093/ecco-jcc/jjw168.

30. Bohl J, Soba K. Indications and options for surgery in ulcerative colitis. Surg Clin N Am. 2015;95(6):1211-32. doi: 10.1016/j.suc.2015.07.003.

31. Bailay $H$, Glasgow S. Challenges in the medical and surgical management of chronic inflammatory bowel disease. Surg Clin N Am. 2015;95(6):1233-44. doi: 10.1016/j. suc.2015.08.003.

32. Gionchetti P, Dignass A, Danese S, et al. 3rd European evidence-based consensus on the diagnosis and management of Crohn's disease 2016: Part 2: Surgical management and special situations. J Crohns Colitis. 2017;11(2):135-49. doi: $10.1093 /$ ecco-jcc/jjw169. 